\title{
DESEMPEÑO ACADÉMICO Y COMPETENCIAS TRANSVERSALES EN ESTUDIANTES DE MEDICINA UNIVERSIDAD DE CARABOBO, VENEZUELA
}

\author{
Everilda Arteaga Navas \\ Gilberto Antonio Bastidas Pacheco \\ Amilcar Josue Pérez Rivero \\ Universidad de Carabobo (Venezuela)
}

\begin{abstract}
RESUMEN: En América Latina el desempeño académico ha mostrado altos índices de deserción, altas tasas de repitencia, elevado número de estudiantes con rezago en sus estudios, bajos promedios de calificaciones y bajas tasas de graduación debido a la desarticulación con el estudiante como persona, específicamente en sus competencias transversales. Estudio descriptivo, correlacional, transversal y de campo. La población fue de 670 estudiantes en partes iguales entre el tercero y sexto año de la Escuela de Medicina de la Universidad de Carabobo, a quienes se les aplicó una encuesta en formato Likert para medir las competencias transversales. Se asumió como muestra $30 \%$ de los estudiantes, lo que implica 100 individuos para cada grupo.

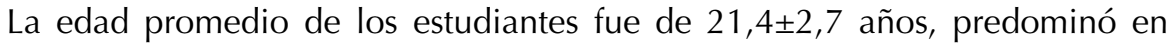
frecuencia el género femenino (62\%) sobre el masculino (38\%), en ambos grupos prevalecieron los estratos socioeconómicos II y III. Las competencias transversales son percibidas como deficientes. En la descripción del desempeño académico y las competencias transversales las relaciones en su mayoría resultaron negativas y de grado muy bajo lo que denota una tendencia inversa. Solo con el desempeño del trabajo y motivación las correlaciones fueron positivas también de grado muy bajo.
\end{abstract}

PALABRAS CLAVE: rendimiento académico, competencia, medicina, docencia, universidad, estudiante, educación superior.

\section{ACADEMIC PERFORMANCE AND TRANSVERSAL COMPETENCES IN MEDICAL STUDENTS, UNIVERSITY OF CARABOBO, VENEZUELA}

\footnotetext{
ABSTRACT: In Latin America, academic performance has shown high dropout rates, high repetition rates, a high number of students lagging behind
} 
in their studies, low grade point averages, and low graduation rates due to disarticulation with the student as a person, specifically in their studies. transversal competences. Descriptive, correlational, cross-sectional and field study. The population was 670 students in equal parts between the third and sixth year of the School of Medicine of the University of Carabobo, to whom a survey in Likert format was applied to measure transversal competences. $30 \%$ of the students were assumed as a sample, which implies 100 individuals for each group. The average age of the students was $21.4 \pm 2.7$ years, the female gender $(62 \%)$ prevailed over the male $(38 \%)$, in both groups socioeconomic strata II and III prevailed. The transversal competences are perceived as deficient. In the description of academic performance and transversal competences, the relationships were mostly negative and of a very low degree, which indicates an inverse trend. Only with work performance and motivation were the correlations positive, also of a very low degree.

KEYWORDS: Academic performance, competition, medicine, teaching, university, student, higher education.

Recibido: 18/01/2021

Aceptado: 01/03/2021

Correspondencia: Gilberto Antonio Bastidas Pacheco, Facultad de Ciencias de la Salud. Universidad de Carabobo, Naguanagua 2005, Carabobo, Venezuela. Email: bastidasprotozoo@hotmail.com

\section{INTRODUCCIÓN}

El desempeño académico es amplio, de gran cobertura y multicausal afectado por cuatro grupos de factores a saber: los fisiológicos relacionados con las condiciones de salud del estudiante; los pedagógicos en mención directa a la calidad de la enseñanza y al aprendizaje (que involucra entre otros el tamaño del curso, los métodos y estrategias utilizadas, el desempeño del docente, los hábitos de estudio y las conductas académicas); los psicológicos que implican las variables de personalidad, motivación, actitud y afectividad de los estudiantes, al igual que las expectativas, vocación y satisfacción; y sociológicas que involucran las condiciones socioeconómicas, familiares y laborales (Romo y fresan, 2000; Krol, Dobson y Adesina, 2019).

En América Latina el desempeño académico ha resultado afectado negativamente (con altos índices de deserción, altas tasas de repitencia, elevado número de estudiantes con rezago en sus estudios, bajos promedios de calificaciones y bajas tasas de graduación) debido (fundamentalmente a partir de la década de los setenta) al crecimiento exponencial en la matrícula de las universidades con las siguientes implicaciones: deficiencias en los recursos financieros y físicos, poca preparación pedagógica y vocación del docente, bajo nivel de salud y nutrición del estudiante, 
inequidades en el acceso a las instituciones universitaria, disminución de la calidad en los procesos educativos con el mantenimiento de la pedagogía tradicional (centrada en la presencia física y memoria) y fuerte desarticulación con el estudiante como persona, pues se abandona al ser (Aldana, Roberti y Rodríguez, 2010).

Los datos permiten constatar que el bajo desempeño académico es un problema alarmante en el conjunto de las universidades que pone en tela de juicio la calidad de las instituciones de educación superior, ya que, no consideran o la hacen parcialmente los aspectos inherentes al hombre en su formación (Yorke, 1998; Cabrera, Álvarez y González, 2006). Este es un tema que preocupa en lo económico a la universidad y a sus estudiantes por el gran coste social que supone el mantenerlos hasta graduarlos en el tiempo establecido, coste que se magnifica cuando el estudiante cursa estudios por más tiempo al formalmente establecido o abandona su carrera (Cabrera, Álvarez y González, 2006).

Si bien esta situación es perturbadora existen hallazgos que reflejan que los estudiantes que abandonan por bajo desempeño académico tienen la intención de completar sus estudios universitarios, por lo que puede tratarse no simplemente de deserción universitaria o prolongación de estudios, sino de fracaso vocacional o académico inducido posiblemente por trastornos o fallas en las competencias (Cabrera, Álvarez y González, 2006; Sastre-Fullana, De Pedro-Gómez, Bennasar-Veny, Serrano-Gallardo y Morales-Asencio, 2014). Desde esta perspectiva el significado de cada situación puede variar y por tanto merece particular estudio.

Ahora bien es pobre la preocupación latinoamericana y particularmente la venezolana por el estudio del despeño académico, pues la mayoría de las investigaciones se han centrado en aspectos como: rendimiento estudiantil aplicado a una asignatura particular, aprendizaje cooperativo, variables motivacionales, programas para mejorar la comprensión lectora, estrategias didácticas mediadoras, tutoría del preparador docente, estrategias de evaluación, entre otras, en consecuencia el desempeño se interpreta solo con base en calificaciones alcanzadas en obvio desconocimiento de la persona como ser.

El éxito académico de los estudiantes está también ligado a las competencias transversales, es decir, a la capacidad para realizar cualquier tarea que le asegure la adquisición de conocimientos y le permita regular su propio aprendizaje (solos o en grupos) en plena conciencia de los procesos de pensamiento (los propios), de las estrategias y de los métodos que se usan para aprender (OCDE, 2003), en pocas palabras las competencias transversales o capacidad para "aprender a aprender" son un prerrequisito para el éxito académico (Bravo, 2005; Soler-González, But, Boada, Ayala, Peñascal, Rodríguez y GERDS group, 2016).

En este sentido se sabe de estudiantes que desarrollan habilidades genéricas de aprendizaje durante su paso por la educación primaria y secundaria tradicional, pero otros no, es por ello que se justifica su investigación en las aulas universitarias con el fin de establecer programas o políticas a favor de la adquisición o mejoramiento, según el caso, de habilidades genéricas que primero deben ser metacognitivas y luego transferidas a diversas áreas del conocimiento (Anderson, 1967; Alkalay y Dolev, 2019).

Debido a que el desempeño académico es considerado un fenómeno educativo complejo e integral porque involucra al estudiante con sus logros y experiencias, y a las 
diversas dimensiones institucionales que influyen en la iniciación y formación integral de estos sujetos en profesionales pensantes y críticos (Sarwar, Aleem y Nadeem, 2019), es que se hace prudente el estudio de los procesos formativos de la esfera personal especialmente en la carrera de medicina la encargada de moldear profesionales humanitarios con vocación y técnicamente capacitados para proteger y restaurar la salud en un mundo globalizado y despersonalizado (Olenick, Flowers, Maltseva y Diez-Sampedro, 2019), y puesto que para Venezuela y específicamente para los estudiantes de la carrera de medicina no se tiene información sobre competencia y su influencia en el desempeño académico se pretende desde la Universidad de Carabobo indagar al respecto con el objeto de aportar información a las autoridades universitarias y del Estado venezolano para la toma de decisiones en procura de potenciar la formación estudiantil.

\section{Metodología}

Estudio descriptivo, correlacional, transversal y de campo. La población estudiada fueron 670 estudiantes a partes iguales entre el tercero y sexto año de la Escuela de Medicina, Sede Carabobo de la Facultad de Ciencias de la Salud de la Universidad de Carabobo, a quienes se les aplicó dos encuestas: una para medir las competencias transversales y la otra el nivel socioeconómico. Debido a la ausencia en Venezuela de estudios previos, se asumió como muestra 30\% de los estudiantes, con un nivel de confiabilidad 95\%, lo que implica un $\mathbf{n}$ muestral de 200 estudiantes, 100 para cada año de la carrera.

\subsection{Técnicas e instrumentos de recolección de datos}

Para escoger la muestra se efectuó la selección probabilística por cuotas respetando la proporción de estudiantes en relación al total. Al interior del grupo se empleó el método de la tómbola para efectuar la escogencia particular. Antes de la aplicación del instrumento se obtuvo el consentimiento informado de cada participante, previa explicación de los objetivos de la investigación. Con el fin de determinar la confiabilidad del instrumento se aplicó una prueba piloto a una submuestra equivalente a la muestra ideal y para la evaluación se empleo el método Alfa de Cronbach.

De la misma manera se recogieron datos respecto a edad, género y nivel socioeconómico. La Dirección de Asuntos Estudiantes de la Universidad de Carabobo proporcionó el indicador rendimiento de la dimensión desempeño académico de los estudiantes participantes. El rendimiento académico se constituye en un indicador del nivel de aprendizaje alcanzado por el estudiante, representa la eficacia en la consecución de los objetivos curriculares para las diversas asignaturas, y en Venezuela se expresa mediante un calificativo o promedio ponderado basado en el sistema vigesimal (como resultado de las evaluaciones continuas y se constituyen en la tasa de éxito); es decir, las notas varían de 0 a 20 puntos, donde el puntaje aprobatorio es igual o mayor de 10 .

\subsection{Instrumento}

El cuestionario para la autoevaluación de competencias transversales (Solanes, Núñez y Rodríguez, 2008) consta de 45 ítems con una consistencia interna igual a 
0,92, distribuidos en seis factores que explican 53,15\% de la varianza total, en formato Likert con seis categorías de respuesta: (1) Casi nunca, (2) Ocasionalmente, (3) Ordinariamente, (4) Con frecuencia, (5) Muchísimas veces y (6) Siempre, en la tabla 1 se muestra la variable competencias transversales con sus dimensiones, indicadores e ítems. El enunciado de los ítems y el formato del cuestionario siguieron las directrices marcadas por Haladyna y Downing (1989) y Haladyna, Downing y Rodríguez (2002). No se estableció límite de tiempo para responder el cuestionario, dependió del tiempo de ejecución de cada participante. Se realizó operacionalización de las variables (tabla 2 ).

Tabla 1. Variable competencias transversales, sus dimensiones, indicadores e ítems

\begin{tabular}{|c|c|c|}
\hline Dimensiones & Indicadores & $N^{\circ}$ de ítems \\
\hline \multirow[t]{2}{*}{ Instrumental } & Desempeño del trabajo & $\begin{array}{l}3-13-15-16-34-37-39-41-42-43- \\
44-45\end{array}$ \\
\hline & Habilidades para la gestión & 6-7-17-18-19-33-36 \\
\hline Interpersonales & $\begin{array}{l}\text { Relaciones interpersonales y trabajo } \\
\text { en equipo }\end{array}$ & $20-21-22-26-29$ \\
\hline \multirow{3}{*}{ Sistémica } & Liderazgo & $8-10-11-12-14-24-25-27-28-30$ \\
\hline & Motivación & $1-2-5-9-31-35-38$ \\
\hline & Capacidad de aprendizaje & $4-23-32-40$ \\
\hline
\end{tabular}

Fuente: Adaptado de Solanes, Nuñez, Rodríguez (2008).

Tabla 2. Variables cualitativas de la investigación

\begin{tabular}{|c|c|c|c|c|}
\hline Objetivo específico & Variable & Dimensión & Indicador & No Ítem \\
\hline \multirow{6}{*}{$\begin{array}{l}\text { Identificar aspectos } \\
\text { de las competencias } \\
\text { transversales } \\
\text { en estudiantes } \\
\text { de la carrera de } \\
\text { Medicina- Sede } \\
\text { Carabobo, de la } \\
\text { Universidad de } \\
\text { Carabobo. }\end{array}$} & \multirow{6}{*}{$\begin{array}{l}\text { Competencias } \\
\text { transversales }\end{array}$} & Instrumental & $\begin{array}{l}\text { Desempeño del } \\
\text { trabajo }\end{array}$ & $\begin{array}{l}3-13-15-16-34- \\
37-39-41-42-43- \\
44-45\end{array}$ \\
\hline & & \multirow[t]{2}{*}{ Interpersonal } & $\begin{array}{l}\text { Relaciones } \\
\text { interpersonales } \\
\text { y trabajo en } \\
\text { equipo } \\
\end{array}$ & $20-21-22-26-29$ \\
\hline & & & $\begin{array}{l}\text { Habilidades para } \\
\text { la gestión }\end{array}$ & $\begin{array}{l}\text { 6-7-17-18-19- } \\
33-36\end{array}$ \\
\hline & & \multirow{3}{*}{ Sistémica } & Liderazgo & $\begin{array}{l}8-10-11-12-14- \\
24-25-27-28-30\end{array}$ \\
\hline & & & Motivación & $1-2-5-9-31-35-38$ \\
\hline & & & $\begin{array}{l}\text { Capacidad de } \\
\text { aprendizaje }\end{array}$ & $4-23-32-40$ \\
\hline
\end{tabular}

Fuente: Elaborado por los investigadores. 


\subsection{Técnicas de procesamiento y análisis de datos}

Con la información obtenida se efectuaron estadísticas descriptivas como: medidas de tendencia central y dispersión (promedios e intervalos de confianza del 95\% [IC95\%]) y distribuciones de frecuencia. Para probar la dependencia entre los factores antropológicos y el desempeño académico se emplearon las pruebas $x^{2}$, coeficiente de correlación de Pearson, prueba t para grupos independientes y análisis de varianza. Se aceptaron como significativas las pruebas con $\alpha \leq 0.05$. El análisis estadístico se realizó con el programa estadístico SPSS 20.0.

\section{Resultados}

La muestra estuvo conformada por 200 estudiantes distribuida a partes iguales entre cursantes del tercer y sexto año de la carrera de medicina. La edad promedio fue de 21,4 $\pm 2,7$ años, el estudiante de menor edad tenía 18 años y el de mayor 42, fueron en total 124 mujeres (62\%) y 76 hombres (38\%). En los estudiantes del tercer año los que tenían 20 o menos años ( $38 \%=76$ casos) fueron más, de la misma manera el género femenino prevaleció sobre el masculino con 62 casos (31\%) y en relación con el estrato socioeconómico predominaron el II $(20,5 \%=41$ casos) y III $(19 \%=38$ casos). De los estudiantes del sexto año fueron más frecuentes aquellos con más de 20 años $(49,50 \%=99$ casos $)$, del género femenino $(31 \%=62$ casos $)$ e igualmente de los estratos socioeconómicos II $(23 \%=46$ casos $)$ y del III $(14 \%=28$ casos $)$ (tabla 3$)$. El promedio de calificaciones fue similar para ambos géneros y años de la carrera de medicina evaluados, en el tercer año fue de 14,8 para las mujeres y de 14,7 para los hombres, y en sexto año fue de 15,2 y 15,9 respectivamente (tabla 4).

Tabla 3. Edad, género y estrato socioeconómico de estudiantes del tercero y sexto año de la carrera de medicina de la sede Carabobo. Universidad de Carabobo. Venezuela

\begin{tabular}{ccccccc}
\hline Año & \multicolumn{2}{c}{ 3er } & \multicolumn{2}{c}{ 6to } & \multicolumn{3}{c}{ Total } \\
\hline Edad & $\mathbf{f}$ & $\mathbf{\%}$ & $\mathbf{f}$ & $\mathbf{\%}$ & $\mathbf{f}$ & $\mathbf{\%}$ \\
\hline$\leq 20$ años & 76 & 38 & 1 & 0,5 & 77 & 38,5 \\
\hline$>20$ años & 24 & 12 & 99 & 49,5 & 123 & 61,5 \\
\hline Género & $\mathbf{f}$ & $\mathbf{\%}$ & $\mathbf{f}$ & $\mathbf{\%}$ & $\mathbf{f}$ & $\mathbf{\%}$ \\
\hline Femenino & 62 & 31 & 62 & 31 & 124 & 62 \\
\hline Masculino & 38 & 19 & 38 & 19 & 76 & 38 \\
\hline Estrato socioeconómico & $\mathbf{f}$ & $\mathbf{\%}$ & $\mathbf{f}$ & $\mathbf{\%}$ & $\mathbf{f}$ & $\mathbf{\%}$ \\
\hline I & 13 & 6,5 & 18 & 9 & 31 & 15,5 \\
\hline II & 41 & 20,5 & 46 & 23 & 87 & 43,5 \\
\hline III & 38 & 19 & 28 & 14 & 66 & 33 \\
\hline IV & 5 & 2,5 & 8 & 4 & 13 & 6,5 \\
\hline V & 3 & 1,5 & 0 & 0 & 3 & 1,5 \\
\hline Total & $\mathbf{1 0 0}$ & $\mathbf{5 0}$ & $\mathbf{1 0 0}$ & $\mathbf{5 0}$ & $\mathbf{2 0 0}$ & $\mathbf{1 0 0}$ \\
\hline
\end{tabular}

Fuente: Datos propios de la investigación. 
Tabla 4. Promedio de calificaciones y género de estudiantes del tercero y sexto año de la carrera de medicina de la sede Carabobo. Universidad de Carabobo. Venezuela

\begin{tabular}{ccccccccc}
\hline Año & \multicolumn{9}{c}{ 3er } & \multicolumn{3}{c}{ 6to } \\
\hline Género & Promedio & DS & Max & Min & Promedio & DS & Max & Min \\
\hline Femenino & 14,8 & 1,6 & 17,8 & 10,7 & 15,2 & 1,5 & 18,0 & 12,8 \\
\hline Masculino & 14,7 & 1,4 & 17,5 & 11,7 & 15,9 & 1,6 & 18,4 & 12,4 \\
\hline
\end{tabular}

Fuente: Datos propios de la investigación.

El estudio del desempeño del trabajo de la competencia transversal instrumental en estudiantes de la carrera de medicina de la Universidad de Carabobo respecto a las demás categorías de las variables incluidas en la investigación quedó por debajo de los 30 puntos (en consideración que la máxima puntuación de esta dimensión se ubicó en 60) esto es consistente con la tendencia no muy favorable en cuanto a los puntajes originales registrados. Únicamente hubo diferencia significativa en los promedios entre géneros del desempeño del trabajo $(\mathrm{P}<0.05)$, pues fue mayor en las mujeres. Igual panorama, el de poco favorable, se descubrió en las habilidades para la gestión de la competencia transversal instrumental porque los promedios estuvieron por debajo de 17 puntos (tomando en cuenta que la máxima puntuación en esta dimensión fue de 35). El promedio fue levemente mayor en estudiantes con más de 20 años de edad ubicados en tercero (exclusivamente) y en mujeres $(P<0.05)$ (tabla 5$)$.

Tabla 5. Promedios de competencia transversal instrumental según las variables estudiadas en cursantes de la carrera de medicina de la sede Carabobo. Universidad de Carabobo. Venezuela

\begin{tabular}{|c|c|c|c|c|}
\hline \multicolumn{5}{|c|}{ Desempeño del Trabajo } \\
\hline Edad (años) & $\mathbf{n}$ & $\bar{X} \pm E s$ & $\mathbf{t}$ & $P$ valor \\
\hline$\leq 20$ años & 77 & $28,7+/-1,7$ & \multirow{2}{*}{0,259} & \multirow{2}{*}{0.7953} \\
\hline$>20$ años & 123 & $28,4+/-1,4$ & & \\
\hline Género & $\mathbf{n}$ & $\bar{X} \pm E s$ & $\mathbf{t}$ & P valor \\
\hline Femenino & 124 & $29,6+/-1,4$ & \multirow{2}{*}{2,699} & \multirow{2}{*}{$0.0075^{*}$} \\
\hline Masculino & 76 & $26,7+/-1,7$ & & \\
\hline Año de la carrera & $\mathbf{n}$ & $\bar{X} \pm E s$ & $\mathbf{t}$ & P valor \\
\hline 3er año & 100 & $28,9+/-1,6$ & \multirow{2}{*}{0,737} & \multirow{2}{*}{0.4616} \\
\hline $6^{\circ}$ año & 100 & $28,1+/-1,5$ & & \\
\hline \multicolumn{5}{|c|}{ Habilidades para la gestión } \\
\hline Edad (años) & $\mathbf{n}$ & $\bar{X} \pm E s$ & $\mathbf{t}$ & $P$ valor \\
\hline$\leq 20$ años & 77 & $15,9+/-1,0$ & \multirow{2}{*}{$-0,041$} & \multirow{2}{*}{0.9667} \\
\hline$>20$ años & 123 & $15,9+/-0,8$ & & \\
\hline
\end{tabular}




\begin{tabular}{|c|c|c|c|c|}
\hline Género & $\mathbf{n}$ & $\bar{X} \pm E s$ & $t$ & P valor \\
\hline Femenino & 124 & $16,8+/-0,8$ & \multirow{2}{*}{3,82} & \multirow{2}{*}{$0.0002 *$} \\
\hline Masculino & 76 & $14,4+/-1,0$ & & \\
\hline Año de la carrera & $\mathbf{n}$ & $\bar{X} \pm E s$ & $t$ & P valor \\
\hline 3er año & 100 & $16,1+/-0,9$ & \multirow{2}{*}{0,689} & \multirow{2}{*}{0.4916} \\
\hline $6^{\circ}$ año & 100 & $15,7+/-0,9$ & & \\
\hline
\end{tabular}

Fuente: Datos propios de la investigación.

En la competencia transversal interpersonal los promedios registrados para las variables estudiadas estuvieron por debajo de 11 puntos (donde la máxima puntuación en esta dimensión fue de 25) lo que insinúa una tendencia poco favorable, aunque no puede establecerse una significativa diferencia $(P>0.05)$ los estudiantes mayores de 20 años, del género femenino y del 3er año de la carrera registraron los mayores promedios (tabla 6).

Tabla 6. Promedios de competencia transversal interpersonal según las variables estudiadas en cursantes de la carrera de medicina de la sede Carabobo. Universidad de Carabobo. Venezuela

\begin{tabular}{|c|c|c|c|c|}
\hline \multicolumn{5}{|c|}{ RELACIONES INTERPERSONALES Y TRABAJO EN EQUIPO } \\
\hline Edad (años) & $\mathbf{n}$ & $\bar{X} \pm E s$ & $\mathbf{t}$ & P valor \\
\hline$\leq 20$ años & 77 & $9,7+/-0,8$ & $-1,026$ & 0.3059 \\
\hline$>20$ años & 123 & $10,2+/-0,7$ & & \\
\hline Género & $\mathbf{n}$ & $\bar{X} \pm E s$ & $\mathbf{t}$ & P valor \\
\hline Femenino & 124 & $10,2+/-0,7$ & 1,109 & 0.26846 \\
\hline Masculino & 76 & $9,6+/-0,8$ & & \\
\hline Año de la carrera & $\mathbf{n}$ & $\bar{X} \pm E s$ & t & P valor \\
\hline 3er año & 100 & $10,0+/-0,8$ & 0,019 & 0.9846 \\
\hline $6^{\circ}$ año & 100 & $9,9+/-0,7$ & & \\
\hline
\end{tabular}

Fuente: Datos propios de la investigación.

En la medición de las competencias transversales sistémicas los promedios de liderazgo rondaron los 25 puntos (la máxima puntuación en esta dimensión fue de 50) esto es poco favorable respecto a los puntajes originales registrados para los estudiantes y con diferencia significativa entre géneros (a favor de las mujeres) $(\mathrm{P}<0.05)$. Además, los promedios fueron mayores en estudiantes con 20 o menos años del tercero de la carrera de medicina y del estrato socioeconómico III.

Ahora bien las respuestas a las preguntas sobre la motivación (de las competencias transversales sistémicas) en ambos grupos de estudiantes, es decir, los de tercero y los de sexto año, no parecen variar en función de ninguna de las otras variables estudiadas (edad, género y estrato socioeconómico). En la motivación los promedios 
de las variables incluidas en el estudio fueron menores de 16 puntos (la máxima puntuación en esta dimensión fue 35) lo que indica una tendencia poco favorable en cuanto a los puntajes originales registrados.

En la capacidad de aprendizaje de las competencias transversales sistémicas los promedios se ubicaron por debajo de 10 puntos (tomando en cuenta que la máxima puntuación en esta dimensión fue 20) con una tendencia poco favorable y con diferencia significativa entre género a predominio de las mujeres $(P<0.05)$ (tabla 7$)$.

Tabla 7. Promedios de competencia transversal sistémica según las variables estudiadas en cursantes de la carrera de medicina de la sede Carabobo. Universidad de Carabobo. Venezuela

\begin{tabular}{|c|c|c|c|c|}
\hline \multicolumn{5}{|c|}{ Liderazgo } \\
\hline Edad (años) & $\mathbf{n}$ & $\bar{X} \pm E s$ & $\mathbf{t}$ & P valor \\
\hline$\leq 20$ años & 77 & $26,3+/-1,5$ & \multirow{2}{*}{1,945} & \multirow{2}{*}{0.0531} \\
\hline$>20$ años & 123 & $24,4+/-1,1$ & & \\
\hline Género & $\mathbf{n}$ & $\bar{X} \pm E s$ & $\mathbf{t}$ & P valor \\
\hline Femenino & 124 & $26,0+/-1,2$ & \multirow{2}{*}{2,32} & \multirow{2}{*}{0.0211} \\
\hline Masculino & 76 & $23,8+/-1,4$ & & \\
\hline Año de la carrera & $\mathbf{n}$ & $\bar{X} \pm E s$ & $\mathbf{t}$ & P valor \\
\hline 3er año & 100 & $26,0+/-1,3$ & \multirow{2}{*}{1,98} & \multirow{2}{*}{0.0486} \\
\hline $6^{\circ}$ año & 100 & $24,2+/-1,2$ & & \\
\hline \multicolumn{5}{|c|}{ Motivación } \\
\hline Edad (años) & $\mathbf{n}$ & $\bar{X} \pm E s$ & $\mathbf{t}$ & P valor \\
\hline$\leq 20$ años & 77 & $14,7+/-1,0$ & \multirow{2}{*}{0,506} & \multirow{2}{*}{0.6128} \\
\hline$>20$ años & 123 & $14,4+/-0,6$ & & \\
\hline Género & $\mathbf{n}$ & $\bar{X} \pm E s$ & $t$ & P valor \\
\hline Femenino & 124 & $14,8+/-0,7$ & \multirow{2}{*}{1,32} & \multirow{2}{*}{0.1872} \\
\hline Masculino & 76 & $14,1+/-0,9$ & & \\
\hline Año de la carrera & $\mathbf{n}$ & $\bar{X} \pm E S$ & $\mathbf{t}$ & P valor \\
\hline 3er año & 100 & $14,9+/-0,9$ & \multirow{2}{*}{1,22} & \multirow{2}{*}{0.2253} \\
\hline $6^{\circ}$ año & 100 & $14,2+/-0,6$ & & \\
\hline \multicolumn{5}{|c|}{ Capacidad de aprendizaje } \\
\hline Edad (años) & $\mathbf{n}$ & $\bar{X} \pm E s$ & $\mathbf{t}$ & P valor \\
\hline$\leq 20$ años & 77 & $9,2+/-0,5$ & \multirow{2}{*}{$-0,60$} & \multirow{2}{*}{0.5492} \\
\hline$>20$ años & 123 & $9,4+/-0,4$ & & \\
\hline Género & $\mathbf{n}$ & $\bar{X} \pm E s$ & $\mathbf{t}$ & P valor \\
\hline Femenino & 124 & $9,7+/-0,4$ & \multirow{2}{*}{2,715} & \multirow{2}{*}{0.0072} \\
\hline Masculino & 76 & $8,8+/-0,5$ & & \\
\hline
\end{tabular}




\begin{tabular}{ccccc}
\hline Año de la carrera & $\mathbf{n}$ & $\bar{X} \pm E \boldsymbol{S}$ & $\mathbf{t}$ & P valor \\
\hline 3er año & 100 & $9,5+/-0,5$ & \multirow{2}{*}{1,105} & 0.2704 \\
\hline $6^{\circ}$ año & 100 & $9,2+/-0,4$ & & \\
\hline
\end{tabular}

Fuente: Datos propios de la investigación.

Las correlaciones de los promedios de calificaciones de los estudiantes de ambos años con el desempeño académico y las competencias transversales resultaron negativas y de grado muy bajo lo que denota la existencia de tendencia inversa, es decir, que a mayor promedio de calificaciones menor puntaje tienen los indicadores de las competencias transversales. Solo con el desempeño del trabajo y motivación las correlaciones fueron positivas pero de grado muy bajo. Ninguna correlación fue significativa $(\mathrm{P}>0.05)$ (tabla 8$)$.

Tabla 8. Correlación entre desempeño académico y competencias transversales respecto al rendimiento académico de los cursantes de la carrera de medicina de la sede Carabobo. Universidad de Carabobo. Venezuela

\begin{tabular}{|l|c|c|c|c|c|c|}
\hline & $\begin{array}{c}\text { Desempeño } \\
\text { del trabajo }\end{array}$ & $\begin{array}{c}\text { Habilidades } \\
\text { para la } \\
\text { gestión }\end{array}$ & Liderazgo & Motivación & $\begin{array}{c}\text { Capacidad de } \\
\text { aprendizaje }\end{array}$ & $\begin{array}{c}\text { Relaciones } \\
\text { interpersonales y } \\
\text { trabajo en equipo }\end{array}$ \\
\hline $\begin{array}{l}\text { Rendimiento } \\
\text { académico }\end{array}$ & 0,06 & $-0,01$ & $-0,009$ & 0,04 & $-0,03$ & $-0,01$ \\
\hline $\begin{array}{l}\text { Grado de } \\
\text { relación }\end{array}$ & Muy baja & Muy baja & Nula & Muy baja & Muy baja & Muy baja \\
\hline P valor & 0.4330 & 0.8345 & 0.8990 & 0.5796 & 0.6819 & 0.8393 \\
\hline
\end{tabular}

Fuente: Datos propios de la investigación.

\section{Discusıón}

Los datos obtenidos en esta investigación, por un lado, descubren a una población estudiantil heterogénea, misma que caracteriza a las complejas universidades públicas en gran parte del mundo, y cuya población estudiantil es joven, de estratos socioeconómicos bajo y medio, y del género femenino, pero con distribución homogénea en el rendimiento académico (Cardozo, Poyeda, Guevara, Ortunio, Loaiza y Torres, 2013); y por otro lado, dan en parte respuestas a las inquietudes y expectativas que se generan y escuchan a diario en la Facultad de Ciencias de la Salud de la Universidad de Carabobo sobre la imperiosa necesidad de conocer el mundo de los jóvenes (el de estudiante universitario) con el fin de comprender mejor las dificultades académicas, de participación y de relación que influyen en su desempeño académico.

Se halló en esta investigación que las competencias transversales (desempeño del trabajo, habilidades para la gestión, relaciones interpersonales, liderazgo, motivación y capacidad de aprendizaje) son percibidas como deficientes en tanto a habilidades genéricas se refiere en el logro del mejor desempeño académico, un motivo más 
de preocupación en al accionar universitario porque en las últimas décadas la gran mayoría de las universidades entre ellas la Carabobo están inmersas en procesos de cambios acelerados para integrarse y adaptarse al contexto de globalización e internacionalización y a nuevas condiciones económicas, sociales y políticas (SánchezLedesma, Juanes, Sáncho, Alonso-Sardón y Goncalves, 2016; Xuan, Xue, Zhang, Luo, Jiang, Qi y Wand, 2019).

En este sentido, para la muestra examinada el desempeño del trabajo (dimensión instrumental) y la motivación (sistémica) tienen correlaciones positivas pero de grado muy bajo. Esto contrasta (la tenencia de competencias) con lo hallado en estudiantes de la Universidad de Talca en quienes se evidencia que están clara y notablemente establecidas las competencias instrumentales (51,2\%) e interpersonales $(35,8 \%)$, y que estas últimas tienen una frecuencia superior a la esperada, pero atribuidas a la suerte, a las aptitudes naturales desarrolladas por los estudiantes o bien a la simple e imperiosa necesidad de adaptarse para sobrevivir en el medio académico y no como parte integral y necesaria en el desempeño de las prácticas profesionales. El contraste también se palpa con las profesionales de enfermería (oficio exclusivamente centrado en la atención directa y personal del paciente), aunque solo para las competencias interpersonales (González y Lobato, 2008; Pigolkin, Lomakin y Leonova, E 2018; Purnell, Johnson, Jones, Calloway, Hammond, Hall y Spadaro, 2019).

Al parecer es módica la motivación que esbozan los estudiantes de la carrera de medicina, a pesar de ser considerada uno de los componentes de apreciación positiva de sí mismo y un criterio central del bienestar que le permite a las personas sentirse bien siendo consciente de sus propias limitaciones (Marina, 2010; Matud, López-Curbelo y Fortes, 2019; Orsini, Binnie y Jerez, 2019). El trabajo en equipo y las relaciones interpersonales no fueron encontradas como importantes en estos estudiantes, a pesar que las personas necesitan mantener relaciones sociales estables (para validar el yo) y tener amigos en los que pueda confiar para evitar la sensación subjetiva de infelicidad que el aislamiento social, la soledad y la pérdida del apoyo humano generan, lo que finalmente se traducirá en descenso de la autopercepción, en experimentación de la pérdida del bienestar personal y en menor desempeño en las actividades académicas (Comer, Schweigerm y Shelton, 2019; Matud, LópezCurbelo y Fortes, 2019 ; Sabato, Pérez, Jiang y Feldman, 2019).

De forma general y respecto a la relación entre desempeño académico (a través del indicador rendimiento académico) y competencias transversales se observó que a mayor promedio de calificaciones, es decir, mayor rendimiento, menor fue el puntaje en los indicadores de las competencias transversales, solo con el desempeño del trabajo (dimensión instrumental) y motivación (sistémica) las correlaciones fueron positivas pero de grado muy bajo. Lo descubierto en cuanto a capacidad en habilidades genéricas es contrario (el que no parezca importante las competencias transversales para mejorar el desempeño académico) a la conceptualización que se tiene de la misma, es decir, el de ser estrategias y operaciones comunes a la mayoría de las profesiones que se relacionan con la puesta en práctica integrada de actitudes, rasgos de personalidad, conocimientos y valores adquiridos para el desarrollo de destrezas técnicas de la carrera y cruciales para el ejercicio de la profesión una vez titulados (Sánchez, Leal, Díaz, Carrillo y Jiménez, 2018). 
Entonces puede señalarse que para la muestra no parece indispensable el saber hacer (capacidad generadora que combina una infinidad de conductas adecuadas a un cúmulo de nuevas tareas), el saber estar y el ser capaz de resolver problemas de forma autónoma y flexible (Castaño, Gallón, Gómez y Vásquez, 2004; González y Lobato, 2008; Duarte, Miura, sawada, Alves y Petrin, 2019). Todo ello a pesar de lo señalado por otros autores en relación con las competencias, quienes afirman que existen vínculos entre las competencias disciplinarias y transversales, porque las competencias transversales se encuentran en la intersección entre las distintas disciplinas. En esta intersección se activan procesos fundamentales del pensamiento que engloban interacciones sociales, cognoscitivas, emocionales, culturales y psicomotoras del estudiante y la realidad que lo envuelve (Shinners y Franqueiro, 2017).

Vale preguntarse a la luz de los resultados obtenidos en la correlación entre competencias transversales y desempeño académico hasta qué punto domina el estudiante del sexto año de la carrera de medicina de la Universidad de Carabobo las competencias para comunicarse eficazmente en forma oral, escrita, gráfica y de manera gestual y corporal; si usa adecuadamente las herramientas metodológicas de construcción del conocimiento; hasta que nivel logra aprendizajes autónomos para la aplicación de metodologías creativas de solución de problema; si está en condiciones de autocrítica y autoestima equilibrada; si puede controlar eficazmente el estrés e interactuar con otros integrado a redes de trabajo y sociales; y si puede desarrollar su proyecto profesional en concordancia con su proyecto de vida (Wiliam, 2011; González, Arquero y Hassall, 2014; Mason, Wentling, Cozzo, George, Hampe, Hellier, Kaufmann, Locasto y McDemott, 2018).

Luego de finalizado este estudio, entre los primeros de su tipo para Venezuela, sobre lo que piensan los estudiantes de su desempeño académico desde la perspectiva de las competencias, en procura de mejorar la comprensión que sobre estos aspectos poseen las autoridades educativas para la implementación de políticas de enseñanza que contribuyan con el éxito académico de quienes estudian medicina en las universidades venezolanas en particular y del mundo en general, queda, sin embargo, aún sobre la mesa de debates las siguientes cuestiones: ¿Cuáles son las competencias transversales necesarias a desarrollar?, ¿En qué ciclo del sistema educativo es necesario implementarlas en el currículo? y ¿De qué manera se pueden desarrollar? (Bravo, 2005), y el Informe PISA (Informe PISA, 2002). Asimismo se citan experiencias particulares de algunos Estados entre los que se encuentran: los trabajos realizados por la Universidades de: Cambridge (Melucci, 1996) y Pompeu Fabra (Mir, 2007).

\section{Conclusiones}

La edad promedio de los estudiantes de medicina fue de $21,4 \pm 2,7$ años, predomino el género femenino (62\%) sobre el masculino (38\%), los estudiantes del tercero en su mayoría tenían 20 o menos años y los de sexto año más de 20, en ambos grupos de estudiantes prevalecieron los estratos socioeconómicos II (43,5\%) y III (23\%). Los estudiantes perciben deficiencia en las competencias transversales instrumentales, sistémicas e interpersonales y de las dimensiones analizadas solo se observaron con diferencias significativas promedios mayores en las mujeres en cuanto al desempeño 
del trabajo (competencia transversal instrumental), en habilidades para la gestión (instrumental), liderazgo (competencia transversal sistémica) y en la capacidad de aprendizaje (sistémica).

A mayor promedio de calificaciones menor fue el puntaje en los indicadores de las competencias transversales, solo con el desempeño del trabajo (instrumental) y motivación (sistémica) las correlaciones fueron positivas pero de grado muy bajo. El desempeño académico en relación con las competencias transversales constituye un tema sobre el que recién comienza a producirse conocimiento especializado. Si bien los temas son susceptibles de contar con un marco teórico-conceptual propio, todavía se requiere de esfuerzos para definir con claridad sus fronteras. Porque plantearse estos temas suponen transitar por su multidimensionalidad y complejidad, por cuanto son términos que abarcan lo cultural, social y lo psicológico. Comprender el fenómeno antropológico del estudiante universitario, enriquecerá los estudios que se han realizado con anterioridad acerca de este tema, y permitirá generar planes educativos de mayor calidad, ofrecer mejores servicios a la comunidad estudiantil y del mismo modo fomentar una formación estudiantil sólida, acorde con la misión, la visión y los valores de la institución de educación superior a la que pertenezcan.

\section{ReFERENCIAS BIBLIOGRÁFICAS}

Aldana, K., de Roberti, P. y Rodríguez, M. (2010). Visión del desempeño académico estudiantil en la Universidad Centroccidental Lisandro Alvarado. Compendium, 24, 5-22. https://dialnet.unirioja.es/descarga/articulo/3424052.pdf

Alkalay, S. y Dolev, A. (2019). Public educational psychology services in Israel on the internet. Israel Journal of Health Policy Research, 8(1), 31. https://doi.org/10.1186/ s13584-019-0298-4

Anderson, R. (1967). Educational psychology.Annual review of developmental psychology, 18, 129-164. https://doi.org/10.1146/annurev.ps.18.020167.001021

Bravo, N. (2005). Competencias Proyecto Tuning-Europa, Tuning-America Latina. http://www.cca.org.mx/profesores/cursos/hmfbcp_ut/pdfs/m1/competencias_proyectotuning.pdf

Cabrera, L., Álvarez, P. y González, M. (2006). El problema del abandono de los estudios universitarios. Relieve, 12(2), 171-203. https://doi.org/10.7203/relieve.12.2.4226

Cardozo, R., Poyeda, J., Guevara, H., Ortunio, M., Loaiza, L. y Torres, E. (2013). Características socio-demográficas de estudiantes de Medicina de dos Universidades Públicas en España y en Venezuela. 2009-2010. Vitae: Academia Biomédica Digital, 54. https://dialnet.unirioja.es/servlet/articulo?codigo=7329745

Castaño, E., Gallón, S., Gómez, K. y Vásquez, J. (2004). Deserción estudiantil universitaria: una aplicación de modelos de duración. Lecturas de Economía, 60, 39-65. http://www.redalyc.org/articulo.oa?id=155217798002

Comer, R., Schweigerm, T. y Shelton, P. (2019). Impact of Students' Strengths, Critical Thinking Skills and Disposition on Academic Success in the First Year of a PharmD Program. The American Journal of Pharmaceutical Education, 83(1), 6499. https:// doi.org/10.5688/ajpe6499 
Duarte, R., Miura, I., Sawada, N., Alves, M. y Petrin, R. (2019). The development of transversal competence of health service managers. Revista de saúde pública, 53, 74. https://doi.org/10.11606/s1518-8787.2019053001292

González, J., Arquero, J. y Hassall, T. (2014). Consolidación de la formación por competencias en la universidad española: Estudio de un caso. Educación XX1, 17(2), 145-168. https://doi.org/10.5944/educxx1.17.2.11483

González, N. y Lobato, C. (2008). Evaluación de las competencias sociales en estudiantes de enfermería. Bordón, 60(2), 91-105. https://recyt.fecyt.es/index.php/ BORDON/article/view/29017/15477

Haladyna, T. y Downing, S. (1989). The validity of a taxonomy of multiplechoice test item. Applied Measurement in Education, 1, 51-78. https://doi.org/10.1207/ s15324818ame0201_4

Haladyna, T., Downing, S. y Rodríguez, M. (2002). A review of multiple-choice item-writing guidelines. Applied Measurement in Education, 15(3), 309-334. https://doi.org/10.1207/S15324818AME1503_5

Informe PISA 2000. (2002). Conocimientos y aptitudes para la vida. https://www. oecd.org/pisa/39817007.pdf

Krol, E., Dobson, R. y Adesina, K. (2019). Association Between Prerequisites and Academic Success at a Canadian University's Pharmacy Program. American Journal of Pharmaceutical Education, 83(1), 6491. https://doi.org/10.5688/ajpe6491

Marina, J. (2010). La competencia de emprender. Revista de Educación, 351, 49-71. https://www.revistaeducacion.educacion.es/re351/re351_03.pdf

Mason, D., Wentling, W., Cozzo, N., George, L., Hampe, H., Hellier, S., Kaufmann, J., Locasto, L. y McDemott, D. (2018). Supporting the Quality of Measurement and Evaluation in Education. Journal of Nuclear Medicine Technology, 46(4), 384-390. https://doi.org/10.2967/jnmt.118.210385

Matud, M., López-Curbelo, M. y Fortes, D. (2019). Gender and Psychological Well-Being. International Journal of Environmental Research and Public Health, 16(19), 3531. https://doi.org/10.3390/ijerph16193531

Melucci, A. (1996). The playing self. person and meaning in the planetary society. Cambridge: Cambridge University Press. Cambridge Cultural Social Studies. https://doi.org/10.1017/CBO9780511520907

Mir, A. (2007). Las competencias transversales en la Universidad Pompeu Fabra. La visión de los docentes y estudiantes de segundo ciclo, Red U. Revista de Docencia Universitaria, 1. https://doi.org/10.4995/redu.2008.6279

OCDE. (2003). Cadre d'evaluation PISA 2003 - Connaissances et compétences en mathématiques. http://www.oecd.org/education/school/programmeforinternationalstudentassessmentpisa/33694924.pdf

Olenick, M., Flowers, M., Maltseva, T. y Diez-Sampedro, A. (2019). Research in Academia: Creating and Maintaining High Performance Research Teams. Nursing Research and Practice, 1-3. https://doi.org/10.1155/2019/8423460

Orsini, C., Binnie, V. y Jerez, O. (2019). Motivation as a Predictor of Dental Students' Affective and Behavioral Outcomes: Does the Quality of Motivation Matter? Journal Dental of Education, 83(5), 521-529. https://doi.org/10.21815/JDE.019.065 
Pigolkin, I., Lomakin, Y. y Leonova, E. (2018). The significance of student competitions for the development of motivation for education and the acquisition of professional competences in the students the Department of Forensic Medical Expertise of the Sechenovsky University]. Sudebno-meditsinskaia ekspertiza, 61(1), 65-67. https://doi.org/10.17116/sudmed201861165-68

Purnell, M., Johnson, M., Jones, R., Calloway, E., Hammond, D., Hall, L. y Spadaro, D. (2019). Spirituality and Religiosity of Pharmacy Students. American Journal of Pharmaceutical Education, 83(1), 6795. https://doi.org/10.5688/ajpe6795

Romo, A. y Fresan, M. (2000). Los factores curriculares y académicos relacionados con el abandono y el rezago. https://bit.ly/2x5cHDX

Sabato, E., Pérez, H., Jiang, S. y Feldman, C. (2019). Elements of Undergraduate Education Related to Students' Academic Performance in the First Year of Dental School. Journal Dental of Education, 83(5), 510-520. https://doi.org/10.21815/ JDE.019.066

Sánchez, J., Leal, C., Díaz, J., Carrillo, M. y Jiménez, D. (2018). Socio-emotional competencies as predictors of performance of nursing students in simulated clinical practice. Nurse Education in Practice, 32, 122-128. https://doi.org/10.1016/j. nepr.2018.07.009

Sánchez-Ledesma, M., Juanes, J., Sáncho, C., Alonso-Sardón, M. y Gonçalves, J. (2016). Acquisition of Competencies by Medical Students in Neurological Emergency Simulation Environments Using High Fidelity Patient Simulators. Journal of Medical Systems, 40(6), 139. https://doi.org/10.1007/s10916-016-0496-3

Sarwar, S., Aleem, A. y Nadeem, M. (2019). Health Related Quality of Life (HRQOL) and its correlation with academic performance of medical students. Pakistan Journal of Medical Sciences, 35(1), 266-270. https://doi.org/10.12669/pjms.35.1.147

Sastre-Fullana, P., De Pedro-Gómez, J., Bennasar-Veny, M., Serrano-Gallardo, P. y Morales-Asencio, J. (2014). Competency frameworks for advanced practice nursing: a literature review. International Nursing Review, 61(4), 534-542. https://doi. org/10.1111/inr.12132

Shinners, J. y Franqueiro, T. (2017). Individual and Collective Competence. Journal of Continuing Education Nursing, 48(4), 148-150. https://doi.org/10.3928/0022012420160321-02

Solanes, A., Núñez, R. y Rodríguez, J. (2008). Elaboración de un cuestionario para la evaluación de competencias genéricas en estudiantes universitarios. Apuntes de Psicología, 26(1), 35-49. http://www.apuntesdepsicologia.es/index.php/revista/ article/view/250/252

Soler-González, J., But, I. M., Boada, J., Ayala, V., Peñascal, E., Rodríguez. y GERDS group. (2016). Do primary health centres and hospitals contribute equally towards achievement of the transversal clinical competencies of medical students? Performance on the Objective Structured Clinical Examination (OSCE) in competency acquisition. Atención Primaria, 48(1), 42-48. https://doi.org/10.1016/j. aprim.2015.02.005

Wiliam, D. (2011). What is assessment for learning? Studies in Educational Evaluation, 37, 3-14. https://doi.org/10.1016/j.stueduc.2011.03.001 
Xuan, X., Xue, Y., Zhang, C., Luo, Y., Jiang, W., Qi, M. y Wang, Y. (2019). Relationship among school socioeconomic status, teacher-student relationship, and middle school students' academic achievement in China: Using the multilevel mediation model. PLoS One, 14(3), e0213783. https://doi.org/10.1371/journal. pone.0213783

Yorke, M. (1998). Non-completion of undergraduate study: some implications for policy in higher education. Journal of Higher Education Policy and Management, 20(2), 189-201. https://doi.org/10.1080/1360080980200206 